\title{
Nearest Neighbor Rules PAC-Approximate Feedforward Networks †
}

\author{
Nageswara S. V. Rao \\ Center for Engineering Systems Advanced Research \\ Oak Ridge National Laboratory \\ Oak Ridge, Tennessee 37831-6364 \\ email: raons@ornl.gov
}

\begin{abstract}
"The submitted manuscript has been authored by a contractor of the U.S. Government under contract No. DE. AC05-96OR22464. Accordingly, the U.S. Government retains a nonexelusive, royalty-free license to publish or reproduce the published form of this contribution, or allow others to do so, for U.S. Government purposes."
\end{abstract}

Submitted to: International Conference on Neural Networks, Washington, D. C., June 3-6, 1996.

\section{DISCLAIMER}

\begin{abstract}
This report was prepared as an account of work sponsored by an agency of the United States Government. Neither the United States Government nor any agency thereof, nor any of their employees, makes any warranty, express or implied, or assumes any legal liability or responsibility for the accuracy, completeness, or usefulness of any information, apparatus, product, or process disclosed, or represents that its use would not infringe privately owned rights. Reference herein to any specific commercial product, process, or service by trade name, trademark, manufacturer, or otherwise does not necessarily constitute or imply its endorsement, recommendation, or favoring by the United States Government or any agency thereof. The views and opinions of authors expressed herein do not necessarily state or reflect those of the United States Government or any agency thereof.
\end{abstract}

†Research sponsored by the Engineering Research Program of the Office of Basic Energy Sciences, of the U.S. Department of Energy, under Contract No. DE-AC05-96OR22464 with Lockheed Martin Energy Research Corp. 



\section{DISCLAIMER}

Portions of this document may be illegible in electronic image products. Images are produced from the best available original document. 



\title{
Nearest Neighbor Rules PAC-Approximate Feedforward Networks
}

\author{
Nageswara S.V. Rao \\ Center for Engineering Systems Advanced Research \\ Oak Ridge National Laboratory \\ Oak Ridge, Tennessee 37831-6364 \\ raons@ornl.gov
}

\begin{abstract}
The problem of function estimation using feedforward neural networks based on an independently and identically generated sample is addressed. The feedforward networks with a single hidden layer of $1 /\left(1+e^{-\gamma z}\right)$-units and bounded parameters are considered. It is shown that given a sufficiently large sample, a nearest neighbor rule approximates the best neural network such that the expected error is arbitrarily bounded with an arbitrary high probability. The result is extendible to other neural networks where the hidden units satisfy a suitable Lipschitz condition. A result of practical interest is that the problem of computing a neural network that approximates (in the above sense) the best possible one is computationally difficult, whereas a nearest neighbor rule is linear-time computable in terms of the sample size.
\end{abstract}

\section{Introduction}

Artificial neural networks have been extensively applied in a number of problems involving function estimation. There seem to be (at least) two analytical issues that are under intense investigation by a number of researchers (Roychowdhury et al. [9]). The first aspect deals with the approximation properties of these networks. The second aspect deals with the computation of the "best" neural network to fit the training data. We address both these issues in a statistical formulation along the lines of Cheng and Titterington [3]. Given independently and identically generated points and the values of an unknown function chosen from a family $\mathcal{F}$, we consider the function estimation using feedforward neural networks. We only consider one of the most applied feedforward networks which consist of a single hidden layer of $1 /\left(1+e^{-\gamma z}\right)$-units. The proposed method is applicable to general feedforward networks such that the transfer functions of hidden nodes satisfy Lipschitz conditions.

We show that given a sufficiently large sample, a nearest neighbor rule approximates the best neural network (which achieves minmum expected error), in terms of arbitrarily bounded expected error, with an arbitrary high probability. This result is achieved by utilizing the Lipschitz properties of these neural networks, and by showing the uniform convergence of the nearest neighbor rule in the family of Lipschitz continuous functions.

Our result indicates that the capabilities of the neural networks of this particular kind can be approximately achieved by nearest neighbor rules (which have been extensively studied, e. g. Fukunaga [4]).

Another aspect - perhaps a more important one from a practical view point - is the lack of algorithms with well-understood finite sample behavior to train a neural network (so that it closely approximates the best possible one). This problem is computationally intractable in many cases [9]. Experimentation with several widely employed gradient search methods, e. g. backpropagation, do not seem to yield very good rate of convergence in a number of applications. The existing guarantees of asymptotic convergence or finite sample results are conditioned on a number of smoothness and/or martingale conditions (White [12], Nedeljkovic [6], Rao et al. [8]), which are difficult to verify in practice. From this perspective, it might be worthwhile in some cases to use the nearest neighbor rule which is linear-time computable in terms of the sample size.

We present the problem formulation in Section 2. In Section 3, we first show some uniform convergence properties of nearest neighbor rules in the class of Lipschitz continuous functions, and then relate this result 
to the approximation of the best possible neural network.

\section{Preliminaries}

We consider a feedforward network with a single hidden layer of $m$ hidden nodes and a single output node. The output of the $j$ th hidden node is $\sigma\left(b_{j}^{T} x+t_{j}\right)$, where $x \in[0,1]^{d}, b_{j} \in \Re^{d}, t_{j} \in \Re, b_{j}^{T} x$ is the scalar product, and $\sigma:[0,1] \mapsto[0,1]$ is called an activation function. The output of the network corresponding to input $x$ is given by

$$
f_{w}(x)=\sum_{j=1}^{m} a_{j} \sigma\left(b_{j}^{T} x+t_{j}\right)
$$

where $a=\left(a_{1}, a_{2}, \ldots, a_{m}\right)$ is the weight vector of the output node, and $w$ is the weight vector of the network consisting of $a, b_{1}, b_{2}, \ldots, b_{m}$ and $t_{1}, t_{2}, \ldots, t_{m}$. We consider neural networks with bounded weights such that $w \in[-W,+W]^{m(d+1)}$ for some fixed positive $W<\infty$. We consider hidden units of the particular form $\sigma(z)=1 /\left(1+e^{-\gamma z}\right)$, for $\gamma, z \in \Re$. Let $\mathcal{F}_{W}$ denote set of all functions implemented by neural networks of the above kind, with fixed $d$ and $m$, and various values of $w$.

A training l-sample of a function $f:[0,1]^{d} \mapsto[0,1]$, chosen from a family $\mathcal{F}$, is given ${ }^{1}$ by $\left(x_{1}, f\left(x_{1}\right)\right.$ ), $\left(x_{2}, f\left(x_{2}\right)\right), \ldots,\left(x_{l}, f\left(x_{l}\right)\right)$ where $x_{1}, x_{2}, \ldots, x_{l}, x_{i} \in[0,1]^{d}$, are independently and identically generated according to an unknown distribution $P_{X}\left(X=[0,1]^{d}\right)$. The function learning problem is to estimate a function $\hat{f}:[0,1]^{d} \mapsto[0,1]$, based on the sample, such that the expected absolute error of $\hat{f}$

$$
\left.\|\hat{f}-f\|_{P} \triangleq \int \mid \hat{f}(x)-f(x)\right) \mid d P_{X}
$$

minimized over a class, $\hat{\mathcal{F}}$, of functions. Let $f_{w}^{*} \in \mathcal{F}_{\mathcal{W}}$ minimize $\|\hat{f}-f\|_{P}$ over all $\hat{f} \in \mathcal{F}_{\mathcal{W}}$, i. e., $f_{w}^{*}$ is the best (not necessarily unique), in the sense of $(2.1)$, possible neural network that can be chosen from $\mathcal{F}_{\mathcal{W}}$. In general, $f_{w}^{*}$ cannot be computed from (2.1) since the function $f$ and the underlying probability distribution are both unknown. Furthermore, since no restrictions are placed on the underlying distribution, it is not always possible to infer $f_{w}^{*}$ (with probability one) based on a finite sample. Consequently, often only an approximation $\hat{f}_{w}$ to $f_{w}^{*}$ is feasible.

We consider conditions under which an approximation $\hat{f}_{w}$ to $f_{w}^{*}$ satisfies

$$
P\left[\left\|\hat{f}_{w}-f_{w}^{*}\right\|_{P}>\epsilon\right]<\delta
$$

for arbitrarily specified $\epsilon$ and $\delta, 0<\epsilon, \delta<1$, where $P=P_{X}^{l}$ is the product measure on the set of all independently and identically distributed $l$-samples. Thus the approximation "error" of $\hat{f}_{w}$ is to be bounded by $\epsilon$ with a probability of $1-\delta$ (given a sufficiently large sample). A special case of this formulation, where $f$ is an indicator function, constitutes a basic version of PAC (probably and approximately correct) learning problem formulated by Valiant [11].

Let $\hat{f}_{\text {emp }}$ minimize the empirical risk function

$$
\|\hat{f}-f\|_{l} \triangleq \frac{1}{l} \sum_{i=1}^{l}\left|\hat{f}\left(x_{i}\right)-f\left(x_{i}\right)\right|
$$

over all $\hat{f} \in \mathcal{F}_{W}$. By making use of the bounded capacity of $\mathcal{F}_{W}$ (or using other characterizations, see Anthony [1], Haussler [5]), one can show that $\hat{f}_{e m p}$ satisfies the condition (2.2) given sufficiently large sample. However, the computational problem of obtaining $\hat{f}_{e m p}$ is riddled with some difficulties. At present, a practical approach is to terminate the training algorithm after certain number of iterations, which results only in an approximation to the required $\hat{f}_{\text {emp }}$. Here we suggest that a nearest neighbor rule could provide such an approximation.

\footnotetext{
${ }^{1}$ The case of bounded $f$ defined on a compact domain $X \subseteq \Re^{d}$ can be handled in a manner similar to here, with the diameter of $X$ and the bound on $f$ appearing in the sample size estimates.
} 


\section{Approximation by Nearest Neighbor Rules}

Given $l$-sample $\left(x_{1}, f\left(x_{1}\right)\right),\left(x_{2}, f\left(x_{2}\right)\right), \ldots,\left(x_{l}, f\left(x_{l}\right)\right)$, for $x_{i} \in[0,1]^{d}, f\left(x_{i}\right) \in[0,1]$, we define the nearest neighbor function, $f_{N N}($.$) , as follows. Let V\left(x_{i}\right)=\left\{x \in[0,1]^{d}:\right.$ for all $\left.j,\left\|x-x_{i}\right\| \leq\left\|x-x_{j}\right\|\right\}$, where $\|$.$\| denotes the Euclidean norm. Thus we have the Voronoi decomposition of [0,1]^{d}$ into $V\left(x_{i}\right)$ 's whose boundaries could have non-empty intersections. Then $f_{N N}(x)=f\left(x_{i}\right)$ for $x \in V\left(x_{i}\right)$ (if $x \in V\left(x_{i}\right) \cap V\left(x_{j}\right)$ then $f_{N N}(x)$ is arbitrarily assigned to $f\left(x_{i}\right)$ or $f\left(x_{j}\right)$ ).

We first show some uniform convergence properties of a nearest neighbor rule in the family of Lipschitz continuous functions (see Appendix for proof).

Theorem 3.1 Let $\mathcal{F}_{L(k)}=\left\{f:[0,1]^{d} \mapsto[0,1]\right\}$ denote the family of Lipschitz continuous functions with the constant $k$, i.e. for every $f \in \mathcal{F}_{L(k)}$, we have $|f(x)-f(y)| \leq k\|x-y\|$ for all $x, y \in[0,1]^{d}$. Given a sample of size at least

$$
\frac{4 k^{2} d^{3} e}{\epsilon^{2}} \ln ^{2}\left(\frac{4 k^{2} d^{3} e}{\delta \epsilon^{2}}\right)+2
$$

we have $P\left[\sup _{f \in \mathcal{F}_{L(k)}}\left\|f_{N N}-f\right\|_{P}>\epsilon\right]<\delta$.

We now estimate a Lipschitz constant for the feedforward neural networks along the lines of Tang and Koehler [10].

Lemma 3.1 The Lipschitz constant for a feedforward network $f(x)=\sum_{j=1}^{m} a_{j} \sigma\left(b_{j}^{T} x+t_{j}\right)$ such that $\sigma(z)=$ $1 /\left(1+e^{-\gamma z}\right)$ is given by $k=\frac{\gamma}{4} \sqrt{m\left[1+(d+1) a^{2}\right]}$, where $a=\max _{i} a_{i}$.

Proof: Let $f(x)=\sum_{j=1}^{m} a_{j} \sigma\left(\sum_{i=1}^{d} b_{j i} x_{i}+t_{j}\right)$. The estimate on the Lipschitz constant can be obtained by maximizing the magnitude of the gradient, $\|\nabla w f\|$. First note that

$$
\frac{\partial \sigma(z)}{\partial z}=\gamma \sigma(z)[1-\sigma(z)] \leq \gamma / 4
$$

since the right hand side is maximized at $\sigma(z)=1 / 2$. The result follows from the following bounds

$$
\begin{gathered}
\frac{\partial f}{\partial a_{j}}=\sigma\left(\sum_{i=1}^{d} b_{j i} y_{i}+t_{j}\right) \leq 1 \\
\frac{\partial f}{\partial b_{j i}}=a_{j} \sigma^{\prime}\left(\sum_{i=1}^{d} b_{j i} y_{i}+t_{j}\right) y_{i} \leq a_{j} \gamma^{2} / 4 \\
\frac{\partial f}{\partial t_{j}}=a_{j} \sigma^{\prime}\left(\sum_{i=1}^{d} b_{j i} y_{i}+t_{j}\right) \leq a_{j} \gamma^{2} / 4
\end{gathered}
$$

Note that there are $m$ terms of first type, $m d$ terms of second type, and $m$ terms of third type. Thus we have $\left\|\nabla_{w} f\right\| \leq \frac{\gamma}{4} \sqrt{m+(d+1) \sum_{j=1}^{m}\left(a_{j}\right)^{2}}$ which is upperbounded by $\frac{\gamma}{4} \sqrt{m\left[1+(d+1) a^{2}\right]}$.

To illustrate the main point, we first consider a particular $\mathcal{F}$ such that each $f \in \mathcal{F}$ can be exactly represented by some neural network from $\mathcal{F}_{\mathcal{W}}$, i. e. $\mathcal{F}=\mathcal{F}_{\mathcal{W}}$. We also show an asymptotic result along the lines of consistency results studied in statistical formulations.

Theorem 3.2 For $\mathcal{F}=\mathcal{F}_{W}$, the nearest neighbor rule approximates the best possible feedforward network $f_{w}^{*}$ such that

$$
P\left[\left\|f_{N N}-f_{w}^{*}\right\|_{P}>\epsilon\right]<\delta
$$

given a sample of size $\frac{\gamma^{2} d^{3} e m\left[1+(d+1) a^{2}\right]}{\epsilon^{2}} \ln ^{2}\left(\frac{\gamma^{2} d^{3} e\left[m\left(1+(d+1) a^{2}\right]\right)}{\delta \epsilon^{2}}\right)+2$ where $a=\max _{i} a_{i}$.

Furthermore, $\left\|f_{N N}-f_{w}^{*}\right\|_{P} \rightarrow 0$ as $l \rightarrow \infty$ with probability one. 
Proof: The first part of the theorem follows from Theorem 3.1 by noting that $\mathcal{F}_{W} \subseteq \mathcal{F}_{\mathcal{L}(\|)}$ where $k$ is given in Lemma 3.1. We now show that $\sum_{l=2}^{\infty} P\left[\left\|f_{N N}-f_{w}^{*}\right\|_{P}>\epsilon\right]<\infty$, for all $\epsilon>0$, which shows the second part by the Borel-Cantelli Lemma [2]. Consider the sample size $l=\frac{c}{\epsilon^{2}} \ln ^{2}\left(\frac{c}{\delta \epsilon^{2}}\right)+2$ where $c=\gamma^{2} d^{3} e m\left[1+(d+1) a^{2}\right]$. Then we have

$$
P\left[\left\|f_{N N}-f_{w}^{*}\right\|_{P}>\epsilon\right]<\frac{c}{\epsilon^{2}} e^{\frac{-\sqrt{1-2 \epsilon}}{\sqrt{\epsilon}}}
$$

which yields

$$
\sum_{l=2}^{\infty} P\left[\left\|f_{N N}-f_{w}^{*}\right\|_{P}>\epsilon\right]=\frac{c}{\epsilon^{2}} \sum_{l=2}^{\infty} e^{\frac{-\sqrt{l-3 e}}{\sqrt{c}}} .
$$

The summation term on the right hand side is upperbounded as follows, for $b=\sqrt{c} / \epsilon$,

$$
\sum_{l=2}^{\infty} e^{\frac{-\sqrt{l-2}}{b}} \leq 1+\int_{0}^{\infty} e^{\frac{-\sqrt{x}}{b}} d x=1+2 b \int_{0}^{\infty} e^{-z} z d z=1+2 b .
$$

The implication of the condition of Theorem 3.2 is that for every $l$-sample there exists a neural network that exactly fits the sample. When this condition is not satisfied, define $\hat{f}_{N N}$ as the nearest neighbor rule based on $\left(x_{1}, f_{w}^{*}\left(x_{1}\right)\right),\left(x_{2}, f_{w}^{*}\left(x_{2}\right), \ldots,\left(x_{l}, f_{w}^{*}\left(x_{l}\right)\right)\right.$.

Theorem 3.3 The nearest neighbor rule $\hat{f}_{N N}$ approximates the best possible feedforward such that

$$
P\left[\left\|\hat{f}_{N N}-f_{w}^{*}\right\|_{P}>\epsilon\right]<\delta
$$

given a sample of size $\frac{\gamma^{2} d^{3} e m\left[1+(d+1) a^{2}\right]}{\epsilon^{2}} \ln ^{2}\left(\frac{\gamma^{2} d^{3} e\left[m\left(1+(d+1) a^{2}\right]\right)}{\delta \epsilon^{2}}\right)+2$ where $a=\max _{i} a_{i}$. Furthermore, for sufficiently large sample and fixed $f$, we have $P\left[\left|\left(\left\|f_{N N}-f\right\|_{P}-\left\|\hat{f}_{N N}-f\right\|_{P}\right)\right|>\epsilon\right]<\delta$.

Proof: The first part follows directly from Theorem 3.2. For the second part define $\mathcal{F}_{\mathcal{N N}}$ to be the union of $f_{N N}$ and the set of all nearest neighbor rules $\hat{f}_{N N}$ corresponding to members of $\mathcal{F}_{W}$. First note that on any given sample, the behavior of $\mathcal{F}_{\mathcal{N N}}$ and $\mathcal{F}_{\mathcal{W}}$ is identical except for one element $f_{N N}$. Along the lines of Theorem 3.1 (see Anthony [1]) we can show the following, for a fixed $f$

$$
P\left[\sup _{g \in \mathcal{F}_{\mathcal{N}}}\left|\left[\|g-f\|_{P}-\|g-f\|_{i}\right]\right|>\epsilon / 2\right] \leq \delta
$$

for sufficiently large sample $l$ where $\|g-f\|_{l}=\sum_{i=1}^{l}\left|g\left(x_{i}\right)-f\left(x_{i}\right)\right|$ (a similar result can also be derived using the finite capacity properties [9] of neural network of the present kind). Thus with probability $1-\delta$ we have the following two conditions simultaneously satisfied:

$$
\begin{aligned}
& \left\|f_{N N}-f\right\|_{P} \leq\left\|f_{N N}-f\right\|_{l}+\epsilon / 2 \\
& \left\|\hat{f}_{N N}-f\right\|_{l} \leq\left\|\hat{f}_{N N}-f\right\|_{P}+\epsilon / 2 .
\end{aligned}
$$

Since $\left\|f_{N N}-f\right\|_{l} \leq\left\|\hat{f}_{N N}-f\right\|_{l}$, we have with probability $1-\delta$,

$$
\left\|f_{N N}-f\right\|_{P} \leq\left\|\hat{f}_{N N}-f\right\|_{t}+\epsilon / 2 \leq\left\|\hat{f}_{N N}-f\right\|_{P}+\epsilon .
$$

Notice that the computation of $\hat{f}_{N N}$ requires the knowledge of $f_{w}^{*}$, whereas $f_{N N}$ can be computed from the sample. In the view of this theorem, $f_{N N}$ approximates $\hat{f}_{N N}$ which in turn approximates $f_{w}^{*}$ in the sense of $\mathrm{Eq}(2.2)$. 


\section{Conclusions}

We showed that a nearest neighbor rule provides an approximation in the sense of expected error to the best possible neural network of a specific, but widely employed, architecture. This result is relevant for applications where an approximation to optimal network is adequate and the computational speed is of main concern. If broader classes of neural networks are considered, it would be interesting to see if a nearest neighbor rule still approximates best neural network in the sense of $\mathbf{E q}(2.2)$. It will also be interesting to see if other well-known estimators based on various kernels, wavelets, regression trees, etc., also approximate the neural networks in the sense described here. In the special case $d=1$, linear approximations are shown to satisfy similar approximation properties [7].

\section{Appendix}

Proof of Theorem 3.1: Let us define

$$
C_{\alpha}=\left\{x_{1}, x_{2}, \ldots, x_{l} \in[0,1]^{J d}: \max _{1 \leq i \leq l} P\left[V\left(x_{i}\right)\right]>\alpha\right\}
$$

Further, let $\bar{D}^{\alpha}\left(x_{i}\right)$ be a ball with smallest radius that contains $V\left(x_{i}\right)$, and has a measure of at least $\alpha$. Let $D^{\alpha}\left(x_{i}\right)$ be a ball centered at $x_{i}$ with smallest radius and has a measure of at least $\alpha$. Since the condition $\max _{1 \leq i \leq l} P\left[V\left(x_{i}\right)\right]>\alpha$ implies the condition $\max _{1 \leq i \leq l} \bar{D}^{\alpha}\left(x_{i}\right)>\alpha$, we have

$$
\begin{aligned}
P\left[C_{\alpha}\right] & \leq l P\left\{x_{1}, x_{2}, \ldots, x_{l} \in[0,1]^{l d}: x_{i} \text { is such that for all } j \neq i, x_{j} \notin \bar{D}^{\alpha}\left(x_{i}\right)\right\} \\
& \leq l P\left\{\left(x_{1}, x_{2}, \ldots x_{l}\right) \in[0,1]^{l d}: x_{i} \text { is such that for all } j \neq i, x_{j} \notin D^{\alpha}\left(x_{i}\right)\right\} \\
& \leq l(1-\alpha)^{l-1} .
\end{aligned}
$$

By choosing $\alpha=\frac{1}{l-1} \ln (l / \delta)$, and using the fact that $(1-\beta / n)^{n}<e^{-\beta}$ this probability is no more than $\delta$.

Let us project the $x_{i}$ 's onto to each axis $j=1,2, \ldots, d$ and identify for each $x_{i}$ the interval $I_{i}^{j}$ that contains the projection of $x_{i}$ and contains all the points of the axis that are closet to the projection of $x_{i}$ than projection of any other point. We classify $x_{i}$ 's into two classes based on the condition $\max _{j}\left|I_{i}^{j}\right|<\frac{\rho}{\sqrt{d} k}$, where $\left|I_{i}^{j}\right|$ is the length of the interval $I_{i}^{j}$. If the condition is satisfied for $x_{i}$, the radius of $\vec{D}\left(x_{i}\right)$ is less than or equal to $\rho / k$, where $\bar{D}\left(x_{i}\right)$ is a ball with smallest radius that contains $V\left(x_{i}\right)$; consequently for this $x_{i}$, we have $\left|f(x)-f_{N N}(x)\right| \leq \rho$. If the condition is not satisfied for $x_{k}$, then for some $j$ we have $\left|I_{k}^{j}\right|>\frac{\rho}{\sqrt{d k}}$, which implies that radius of $\bar{D}\left(x_{i}\right)$ is at least $\frac{\rho}{\sqrt{d} k}$. Since there are no more than $\frac{k d^{3 / 2}}{\rho}$ of $i$ 's with the above condition not satisfied (because for each axis $j$, there are no more than $\frac{k \sqrt{d}}{\rho}$ such $x_{i}$ 's), we have that, with probability at least $1-\delta$,

$$
P\left(\left\{x:\left|f(x)-f_{N N}(x)\right| \geq \rho\right\}\right) \leq \frac{k d^{3 / 2} \alpha}{\rho}=\frac{k d^{3 / 2}}{\rho(l-1)} \ln (l / \delta) .
$$

We now estimate an upperbound on $\left\|f(x)-f_{N N}(x)\right\|_{P}$. By noting that $\left|f(x)-f_{N N}(x)\right| \leq k$, for any $x \in[0,1]$, we have

$$
\begin{aligned}
E\left[\left|f(x)-f_{N N}(x)\right|\right] & \leq \int_{0}^{k} P\left[\left|f(x)-f_{N N}(x)\right|>z\right] d z \\
& \leq \rho+\int_{\rho}^{k} P\left[\left|f(x)-f_{N N}(x)\right|>z\right] d z \\
& \leq \rho+a(\ln k-\ln \rho)
\end{aligned}
$$


where $a=\frac{k d^{3 / 2}}{l-1} \ln \left(\frac{l}{\delta}\right)$. The minima of the right hand side of the above equation is achieved at $\rho=a$, thus

$$
\begin{aligned}
E\left[\mid f(x)-f_{N N}(x) \|\right] & \leq a[\ln e k-\ln \ln \rho] \\
& \leq a\left[\ln e k+\ln \left(\frac{l-1}{k}\right)-\ln \ln \left(\frac{l}{\delta}\right)\right] .
\end{aligned}
$$

The last term in the above equation is positive for $l \geq 2$ and hence can be dropped from the upper bound, and thus we have the upper bound

$$
\frac{k}{l-1} \ln \left(\frac{l}{\delta}\right) \ln \left(\frac{e k(l-1)}{k}\right)
$$

which would be upperbounded by $\epsilon$ under the condition $l>\frac{k d^{3 / 2}}{\epsilon} \ln (l / \delta) \ln \left(\frac{e k(l-1)}{k}\right)+1$. Now by using $\ln (\beta l) \leq \sqrt{\beta l}-1$ for $\beta l>1$ we obtain, for $\beta=\frac{\epsilon^{2}}{4 k^{2} d^{3} e}$, the following sufficient condition

$$
l>\frac{k d^{3 / 2}}{\epsilon}\left[\ln \left(\frac{4 k^{2} d^{3} e}{\epsilon^{2} \delta}\right)-1+\frac{\sqrt{l} \epsilon}{\sqrt{e} 2 k d^{3 / 2}}\right] \ln (e l)+1 .
$$

By using $\ln (e l) \leq \sqrt{e l}$, the above condition is implied by

$$
l \geq \frac{2 k d^{3 / 2} \sqrt{e}}{\epsilon} \ln \left(\frac{4 k^{2} d^{3} e}{\delta \epsilon^{2}}\right) \sqrt{l}+2
$$

which in turn is satisfied under the condition $l \geq \frac{4 k^{2} d^{3} e}{\epsilon^{2}} \ln ^{2}\left(\frac{4 k^{2} d^{3} e}{\delta \epsilon^{2}}\right)+2$ where we have used the fact that the condition $l>b^{2}+2$ implies $l \geq b \sqrt{l}+2$.

\section{Acknowledgements}

This research is sponsored by the Engineering Research Program of the Office of Basic Energy Sciences, of the U.S. Department of Energy, under Contract No. DE-AC05-84OR21400 with Martin Marietta Energy Systems, Inc.

\section{References}

[1] M. Anthony. Probabilistic analysis of learning in artificial neural networks: The PAC model and its variants. NeuroCOLT Technical Report Series NC-TR-94-3, Royal Holloway, University of London, 1994.

[2] P. Billingsley. Probability and Measure. John Wiley and Sons, New York, 1986.

[3] B. Cheng and D. M. Titterington. Neural networks: A review from a statistical perspective. Statistical Science, $9(1): 2-54,1994$.

[4] K. Fukunaga. Introduction to Statistical Pattern Recognition. Academic Press, 1990.

[5] D. Haussler. Decision theoretic generalizations of the PAC model for neural net and other learning applications. Information and Computation, 100:78-150, 1992.

[6] V. Nedeljkovic. A novel multilayer neural networks training algorithm that minimizes the probability of classification error. IEEE Transactions on Neural Networks, 4(4):650-659, 1993.

[7] N. S. V. Rao. Fusion methods for multiple sensor systems with unknown error densities. Journal of Franklin Institute, 1995. to appear.

[8] N. S. V. Rao, V. Protopopescu, R. C. Mann, E. M. Oblow, and S. S. Iyengar. Learning algorithms for feedforward networks based on finite samples. Technical Report ORNL/TM-12819, Oak Ridge National Laboratory, September 1994.

[9] V. Roychowdhury, K. Siu, and A. Orlitsky, editors. Theoretical Advances in Neural Computation and Learning. Kluwer Academmic Pub., 1994.

[10] Z. Tang and G. J. Koehler. Deterministic global optimal FNN training algorithms. Neural Networks, 7(2):301$311,1994$.

[11] L. G. Valiant. A theory of the learnable. Communications of the ACM, 27(11):1134-1142, 1984.

[12] H. White. Some asymptotic results for learning in single hidden layer feedforward network models. Journal of American Statistical Association, 84:1008-1013, 1989. 